\title{
Modelación, simulación y control de procesos de fermentación
}

\author{
Modeling, simulation and control of fermentation processes \\ Nelson Aros ${ }^{1} \quad$ Marcelo Cifuentes $^{1} \quad$ Javier Mardones $^{1}$ \\ Recibido 6 de octubre de 2009, aceptado 19 de mayo de 2011 \\ Received: October 6, 2009 Accepted: May 19, 2011
}

\begin{abstract}
RESUMEN
Se presenta un simulador en ambiente Matlab/Simulink para controlar el proceso de fermentación, diseñando una ley de control invariante, luego un control estabilizante para mantener la planta dentro del sistema de referencia y finalmente un control estabilizante mejorado con la implementación de un controlador difuso. El modelo Monod es utilizado y vía simulación se prueban las estrategias aludidas para controlar la tasa de crecimiento específica de la biomasa. El control estabilizante aplicado es superior a la ley invariante y con el PI difuso se logró mejorar el comportamiento en comparación con el PI tradicional.
\end{abstract}

Palabras clave: Proceso de fermentación, modelo Monod, simulación, control estabilizante, control PI difuso.

\begin{abstract}
We present a simulator in Matlab/Simulink environment to control the fermentation process, designing an invariant control law, after that a stabilizing control to keep the plant within the reference system and finally a stabilizing control improved with the implementation of a fuzzy controller. The Monod model is used and tested via simulation above strategies in order to control the specific growth rate of biomass. The stabilizing control is applied above the invariant law and the fuzzy PI was improved behavior compared with traditional PI control.
\end{abstract}

Keywords: Fermentation process, Monod model, simulation, stabilizing control, fuzzy PI control.

\section{INTRODUCCIÓN}

En [1] se presentan varios modelos de biorreactores, donde se identifica que el problema real está dado en la escasez de medidas en línea. Además, la variable de estado dominante, como la concentración de sustrato en el medio, es muy difícil de medir. Otros problemas son la fuerte incertidumbre paramétrica y la presencia de no-linealidad significativa. Como consecuencia de esto, todos los reguladores aplicables deben utilizar la menor cantidad de medidas posibles y ser absolutamente robustos. La importancia de los diseños presentados en [1] es apoyada por un hecho dominante, existen dos modelos o estructuras estándares que representan por lo menos el 95\% de todos los cultivos puros de interés industrial.

En este trabajo se investiga un modelo de fermentación lo suficientemente robusto para realizar un simulador en ambiente Matlab/Simulink para propósitos de control y, a la vez, desarrollar controladores que sean capaces de regular la tasa de crecimiento específica y aumentar la robustez ante variación de las entradas. Así, se presenta la siguiente estructura: se hace una breve descripción de la problemática general, luego se examina un conjunto de modelos matemáticos para biorreactores y sus funciones cinéticas más representativas, luego se describe el problema de

1 Universidad de La Frontera. Avenida Francisco Salazar 01145, Temuco. Chile. E-mail: naros@ufro.cl 
control, junto con las entradas de control y las salidas medibles en el proceso, posteriormente se aborda el diseño de los controladores, después se discuten las pruebas realizadas con cada uno de los controladores implementados y se entregan las conclusiones generales del trabajo realizado.

\section{MODELADO DE BIORREACTORES}

\section{Modelos fenomenológicos}

El crecimiento de microorganismos o biomasa (como las bacterias y levaduras) procede por el consumo de nutrientes apropiados o sustratos (carbono, nitrógeno, oxígeno), bajo condiciones medioambientales favorables (como la temperatura, $\mathrm{pH}$ y otros), ver [3-4]. Asociadas con el desarrollo celular, pero a menudo procediendo en un rango diferente, están las reacciones enzimo catalizadas en las cuales algunos reactantes son transformados en productos por la acción catalítica de enzimas intracelulares o extracelulares, los llamados metabolitos.

El comportamiento dinámico del crecimiento de una población de microorganismos en un solo sustrato limitante en un reactor de tanque agitado se expresa por ecuaciones que dependen tanto de la acumulación total de la biomasa, acumulación total de sustrato, la concentración de la población microbiana (biomasa) en el reactor y en el afluente, la concentración de sustrato en el afluente, la tasa de flujo en el afluente, así como también la tasa de crecimiento microbial, el coeficiente de rendimiento del consumo de sustrato por la biomasa y el volumen del medio de la especie.

Los modelos que consideran la formación de producto, el crecimiento de microorganismos en biorreactores es acompañado a menudo por la formación de productos (los cuales son solubles en el cultivo o son emitidos en forma gaseosa).

\section{Modelos para control}

Actualmente dos modelos estándares cubren una enorme porción de todas las aplicaciones, correspondientes a cultivos puros. Los modelos presentados en esta sección son: no estructurados y no segregados que representan cultivos puros con un sustrato limitador. También se considera un producto el metabolito de interés o, si existe, un inhibidor (en este caso es un producto que afecta de alguna manera el crecimiento microbiano). Para propósitos de control, solamente el producto del segundo caso debe ser considerado.

Un modelo fenomenológico estándar para expresar el proceso de fermentación lo constituyen los biorreactores de lote alimentado (los procesos para la producción de proteínas unicelulares, de alcohol y de ácido glucónico pertenecen a esta categoría [5-6]).

\section{PROBLEMAS DE CONTROL}

Los modelos de los fermentadores son caracterizados por: Alta dimensión del espacio de estados, múltiples entradas y salidas, disturbios externos, significativa no linealidad e incertidumbre, y sofisticados y múltiples objetivos y crítico desempeño [8]. Los biorreactores que se encuentran son: Batch (biorreactores discontinuos), Chemostat y auxostat (biorreactores continuos) y fed-batch (biorreactores de lote alimentado).

En el modo de alimentación por lote se optimiza el uso del sustrato, para ello es deseable hacer que la biomasa aumente a una tasa de crecimiento específica constante. El esquema básico del biorreactor se muestra en la Figura 1.

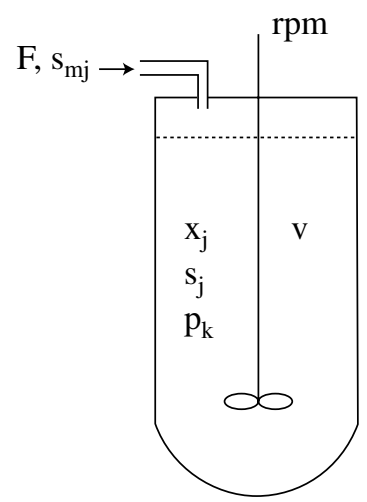

Figura 1. Biorreactor en modo alimentado por lotes.

El modelo del proceso queda dado por:

$$
\begin{aligned}
& \dot{x}=\mu x-D x \\
& \dot{s}=-y_{S} \mu x+D\left(s_{i}-s\right) \\
& \dot{v}=F
\end{aligned}
$$


en donde $x$ corresponde a la biomasa, $s$ la cantidad de sustrato y $v$ volumen del biorreactor. Para obtener un producto deseado se exige el mantenimiento de una tasa de crecimiento específica constante, por esta razón la biomasa y el volumen crecen indefinidamente hasta que no hay más sustrato o se alcance la máxima capacidad del biorreactor.

Puesto que la tasa de crecimiento específica $\mu$ depende de varios factores, la siguiente subespecificación es requerida: las condiciones ambientales se deben mantener constantes en los valores óptimos (como temperatura, $\mathrm{pH}, \mathrm{pO}_{2}$, etc.), dado que en los modelos estándares, $\mu$ puede depender de la concentración de sustrato en el reactor $s$ y de concentraciones de producto $p$. Así, el problema de control, en el caso de $\mu$ constante, implica que el sustrato se mantenga constante, sin embargo el sustrato raramente es medido en línea.

Afortunadamente, es fácil comprobar que la última condición implica trayectorias especiales para las concentraciones de biomasa $x$ y/o el volumen del medio de la especie $v$. Tomando el caso de alimentación por lotes, se puede ver que $\mu$ constante corresponde con una trayectoria exponencial para la cantidad absoluta de biomasa y viceversa. Finalmente, si $\mu$ depende también de $p$ será deseable mantener constante la concentración del producto. Esto, alternadamente, se puede hacer solamente si un sistema está disponible para extraer el producto, es decir, recirculando la parte del caldo a través de un filtro o de un separador.

\section{Entradas de control y perturbaciones}

La cantidad de sustrato provista al biorreactor depende de dos variables: $F$, el flujo de entrada, y $s_{i}$, la concentración de sustrato en el flujo de entrada. Generalmente es solo posible manipular el primero, puesto que no hay actuadores comerciales que permitan variar ambas variables independientemente. Para tener ambas acciones del control disponibles, en la práctica se consideran dos flujos de entradas, $F_{1}$ y $F_{2}$, con concentraciones de sustratos $s_{i n \max } \mathrm{y}$ $s_{\text {in min }}$. El modelo en este caso se presenta a través de las siguientes ecuaciones:

$$
\begin{aligned}
& \dot{x}=\mu x-\left(\frac{F_{1}+F_{2}}{v}\right) x \\
& \dot{s}=-\sigma x+\frac{F_{1}}{v} s_{\text {in } \max }+\frac{F_{2}}{v} s_{\text {in min }}-\left(\frac{F_{1}+F_{2}}{v}\right) s \\
& \dot{p}=\pi x-\left(\frac{F_{1}+F_{2}}{v}\right) p \\
& \dot{v}=F_{1}+F_{2}
\end{aligned}
$$

En cuanto a las perturbaciones, no incluidas en el modelo, las más importantes afectan: al volumen, debido a las muestras para el análisis fuera de línea y la evaporación causada por la aireación, y en algunos casos la concentración de sustrato en el flujo de entrada puede sufrir variaciones.

Medidas disponibles para los biorreactores están dadas en [9]. Para nuestro propósito los puntos principales son: en general, extremadamente difícil medir el sustrato $s$ en línea; existen sensores para la medición de biomasa; también es posible medir el volumen $v$; y otras variables de estado no están disponibles tales como los productos.

Estos sistemas son altamente no lineales, como se aprecia en las funciones cinéticas. También muestran incertidumbre paramétrica y no estructurada. $\mathrm{La}$ incertidumbre dinámica (o no estructurada) es debida al uso de modelos no estructurados y no segregados, así, se ignora parte de la dinámica del sistema, la cual es aglomerada en algunos factores dominantes; y además de las suposiciones tales como homogeneidad o algunas otras simplificaciones del modelo usualmente hechas.

La incertidumbre paramétrica se debe a problemas de identificación; el hecho de que dos poblaciones no sean iguales, debido a efectos ambientales y a la preparación del inóculo, entre otros; el envejecimiento de células que se refleja en variaciones leves de ciertos parámetros durante un experimento. Cualquier cambio en el ambiente o en el caldo puede potencialmente afectar el sistema. Finalmente, hay que considerar la incertidumbre en los actuadores. 


\section{DISEÑO DE CONTROLADORES}

\section{Control invariante}

El caso más simple en el cual el producto no es considerado se representa con el siguiente modelo:

$$
\begin{aligned}
& \dot{x}=\mu(s) x-\frac{F}{v} x \\
& \dot{s}=-y \mu(s) x+\frac{F}{v}\left(s_{i}-s\right) \\
& \dot{v}=F
\end{aligned}
$$

Se sugiere una ley de alimentación exponencial que es expresada como: $F(t)=\lambda x(t) \cdot v(t)=\lambda x_{o} v_{o} e^{\mu_{r} t}$ para algún $\lambda=$ const., donde $x_{o}$ y $v_{o}$ son las condiciones iniciales para la biomasa y el volumen respectivamente, y $\mu_{\mathrm{r}}$ es la tasa de crecimiento específica deseada. Los biotecnólogos saben que en orden a tener una tasa de crecimiento de biomasa constante en una fermentación alimentado por lotes, el flujo de alimentación debería ser "exponencialmente creciente".

\section{Control estabilizador}

El exosistema es un sistema de referencia externo y es utilizado en el diseño de un sistema de control realimentado estabilizador, que contempla un control invariante más dos controladores de la familia PID's, específicamente un controlador PI en la biomasa y un PID para el volumen, ver Figura 2; éstos toman como señal de error las diferencias entre las medidas actuales de las salidas $x, v$ y sus trayectorias de referencia generadas por el exosistema.

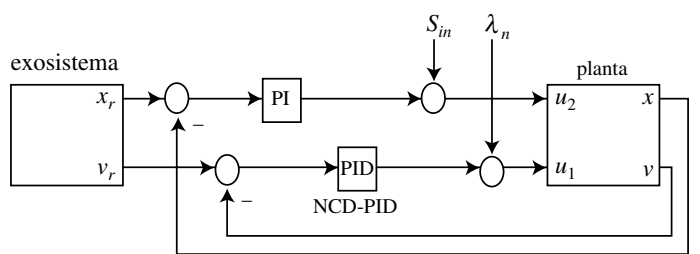

Figura 2. Diagrama en lazo cerrado de control estabilizador.

Tomando el caso más simple en el cual el producto no es considerado y la tasa de crecimiento específica sigue la ley Monod se tiene el siguiente modelo:

$$
\mu=\mu(s)=\frac{\mu_{m} s}{k_{s}+s}
$$

$$
\begin{aligned}
& \dot{x}=\mu x-D x \\
& \dot{s}=-y_{s} \mu x+D\left(s_{i}-s\right) \\
& \dot{v}=F
\end{aligned}
$$

Como $\mu=\mu$ const. y dado que se supone que el modelo es perfecto, no hay perturbaciones y las condiciones iniciales son tal que $s=s_{r}=$ const. $\Rightarrow s=0$. Esta meta se podría alcanzar usando la realimentación parcial del estado: $D=\lambda_{x} \mathrm{o}$ $F=\lambda_{x v}=\lambda x_{m}$, así la ecuación para el sustrato se convierte en $\dot{s}=\left(-y \mu(s)+\lambda\left(s_{i}-s\right)\right) x=0$, con $\lambda$ una constante definida por la expresión:

$$
\lambda=\frac{y_{s} \mu_{r}}{s_{i}-s_{r}}
$$

Sustituyendo en el modelo la ley de realimentación parcial del estado se obtiene el sistema:

$$
\begin{aligned}
& \dot{x}=\mu x-\lambda x^{2} \\
& \dot{s}=\left(-y_{s} \mu+\lambda\left(s_{i}-s\right)\right) x \\
& \dot{v}=\lambda x v
\end{aligned}
$$

Ahora, para superar la incertidumbre del modelo y la presencia de perturbaciones se debe introducir una corrección en la ley de control básica (ley de control invariante). Como se comentó, es interesante el uso de ambas acciones de control $F$ (o equivalentemente $\lambda)$ y $s_{i}$, sin embargo aparece una conexión entre las nuevas acciones de control $u_{1}$ y $u_{2}$ :

$$
\begin{aligned}
& \dot{x}=\mu x-u_{1} x^{2} \\
& \dot{s}=\left(-y_{s} \mu+u_{1}\left(u_{2}-s\right)\right) x \\
& \dot{v}=u_{1} x v
\end{aligned}
$$

Puesto que no hay una medida en línea de la concentración del sustrato $s$ en el biorreactor, una trayectoria exponencial para la cantidad absoluta de la biomasa $x_{m}$ tendrá que ser forzada. Recordando que:

$$
\dot{x}_{m}=\mu(s) \cdot x_{m}
$$

y dado que $x_{m}=x \cdot v$, esta meta se puede alcanzar forzando algunas trayectorias específicas en $x$ y $v$. Para generar esas trayectorias de referencia se usa un exosistema (reemplazando en el modelo $\mu=\mu_{r}=$ const. y $s=s_{r}=$ const $. \Rightarrow \dot{s}=0$ ) se llega a: 


$$
\begin{aligned}
& \dot{x}_{r}=\mu_{r} x_{r}-\lambda_{n} x_{r}^{2} \\
& \dot{v}_{r}=\lambda_{n} x_{r} v_{r}
\end{aligned}
$$

siendo $\lambda_{n}$ una entrada $u_{1}$ nominal determinada usando la expresión $\lambda=\frac{y_{s} \mu_{r}}{s_{i}-s_{r}}$ para una concentración nominal $s_{\text {in }}$ escogida a priori. Así, se obtiene un sistema multivariable (8) con dos entradas y dos salidas. En consecuencia existen dos lazos de control diseñados independientes, es decir sin tomar en cuenta la relación entre $u_{1}$ y $u_{2}$. Con esta meta en mente, y por todo lo dicho acerca de los efectos de cambiar el flujo de entrada y la concentración de sustrato, las siguientes asociaciones son hechas: $u_{1}$ con el volumen $v$ y $u_{2}$ con la concentración de biomasa $x$.

\section{Control estabilizador difuso}

La estructura del controlador difuso incorpora las siguientes partes: Fusificación, base de reglas, motor de inferencia y defusificación.

El regulador difuso implementado es del tipo Mamdani y considera tres variables, dos de entrada y una de salida. Se definieron tres funciones de pertenencia triangulares para las entradas y la salida distribuidas uniformemente en el intervalo [-1,1]. Asimismo, el método de defusificación utilizado para el controlador difuso normalizado es la bisectriz (bisector).

\section{RESULTADOS DE SIMULACIÓN}

\section{Resultados y análisis del control con ley invariante}

Se obtiene como complemento una ley de realimentación exponencial de la forma:

$$
F(t)=\lambda x(t) \cdot v(t)=\lambda x_{o} v_{o} e^{\mu_{r} t}
$$

Con esto se complementa la simulación del proceso que se ha desarrollado utilizando el sistema de ecuaciones (3), con ley de realimentación dada por (4), y cinética de crecimiento específica Monod. En la Figura 3 se muestra el diagrama del proceso implementado en Matlab/Simulink. Para el proceso se usan los datos de planta entregados por [1], ver Tabla 1.
Tabla 1. Parámetros de planta.

\begin{tabular}{|c|c|}
\hline$\mu_{m}$ & 0,22 \\
\hline$y_{s}$ & 1,43 \\
\hline$k_{s}$ & 0,14 \\
\hline
\end{tabular}

Los valores nominales para las entradas $\lambda$ y $s_{i}$ corresponden a $0,0079(l / g h)$ y $20(g / l)$ respectivamente (son entregados en [1]). Las condiciones iniciales para la biomasa, el sustrato y el producto no son entregadas pero fueron aproximadas de tal forma que las curvas resultantes se aproximaran lo mejor posible a las entregadas en [1].

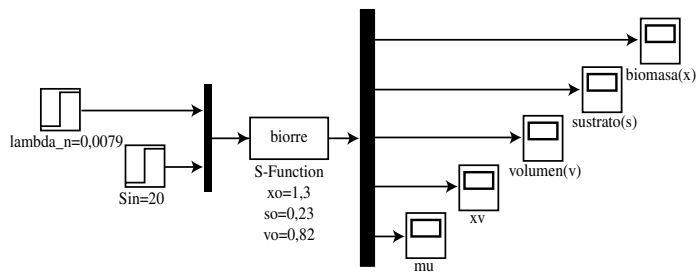

Figura 3. Diagrama en Matlab/Simulink.

La Figura 4 muestra las curvas resultantes para la concentración de la biomasa bajo las entradas a distintos niveles de sustrato y la Figura 5 muestra éstas ante variación de $\lambda$ (las condiciones iniciales de proceso son: $x_{0}=1,3, s_{0}=0,23$ y $v_{0}=0,82$ ). Se aprecia que los resultados son dependientes de las condiciones de la entrada del sustrato y de la variación de $\lambda$.

Resultados y análisis del control estabilizante La Figura 6 muestra el diagrama de bloque del sistema de control estabilizante NCD-PID. Los parámetros de los controladores PI en el lazo de control de volumen y NCD-PID en el lazo de control de biomasa se presentan en la Tabla 2.

Tabla 2. Parámetros del controlador.

\begin{tabular}{|c|c|c|c|}
\hline$\lambda_{n}$ & 0,0079 & $s_{i n}$ & 20 \\
\hline$k_{p 1}$ & 0,1 & $k_{p 2}$ & 150,8 \\
\hline$k_{i 1}$ & $0,1 / 100$ & $k_{i 2}$ & $-1,2471$ \\
\hline$k_{d 1}$ & 0,00 & $k_{d 2}$ & 41,043 \\
\hline
\end{tabular}

Para el sistema con control estabilizante se tienen los siguientes resultados: (i) ante una variación del sustrato de entrada, en la Figura 7 se aprecia que la concentración de la biomasa llega a su término siempre al mismo tiempo, no hay una desviación excesiva en el tiempo de término del proceso; y 


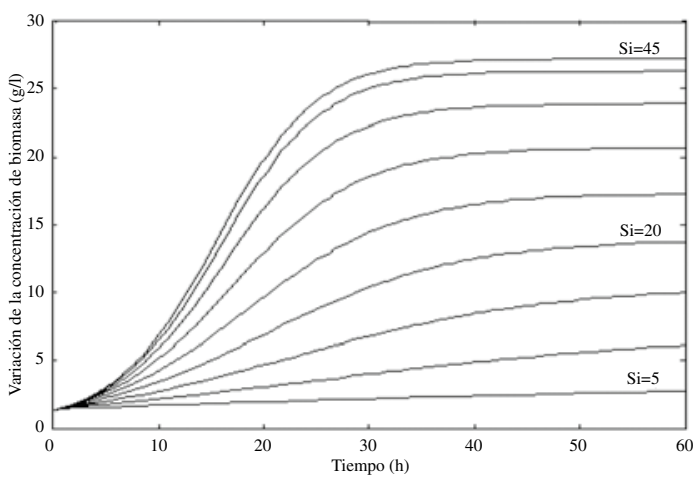

Figura 4. Variación de la concentración de la biomasa ante cambios en la entrada de sustrato, $S_{i}[\mathrm{~g} / \mathrm{l}]$.

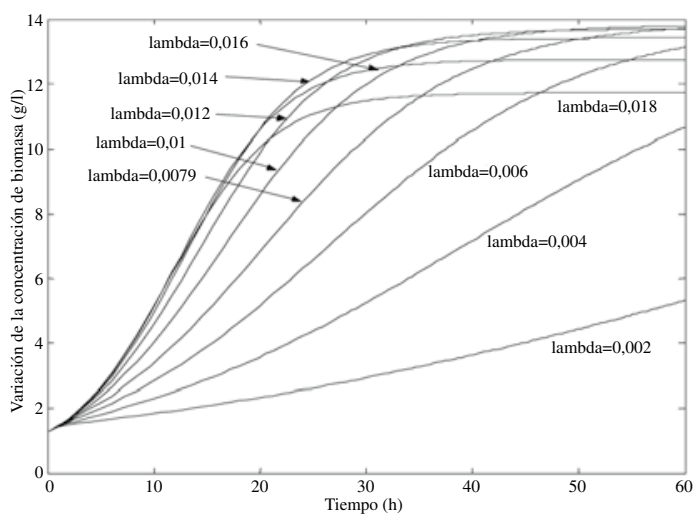

Figura 5. Variación de la concentración de la biomasa ante cambios en la entrada $\lambda$.

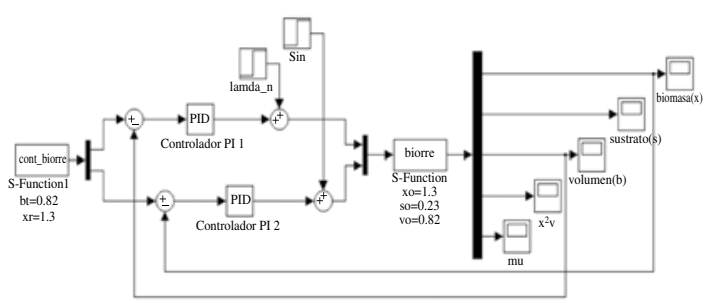

Figura 6. Diagrama de control estabilizante NCDPID.

en la Figura 8 se muestra el comportamiento de la velocidad de crecimiento, donde se aprecia que dependiendo del sustrato de entrada la velocidad sufre un transiente antes de llegar al estado de velocidad constante de crecimiento; (ii) ante variación de $\lambda$ de entrada, en la Figura 9 se muestra el comportamiento de la concentración de la biomasa, se aprecia una leve variación al comienzo de la fermentación debido a que la velocidad de crecimiento no es constante, como se observa en la Figura 10. Así, se puede concluir que cuando la tasa de crecimiento se mantiene dentro de un rango aceptable, el sistema de control es robusto.

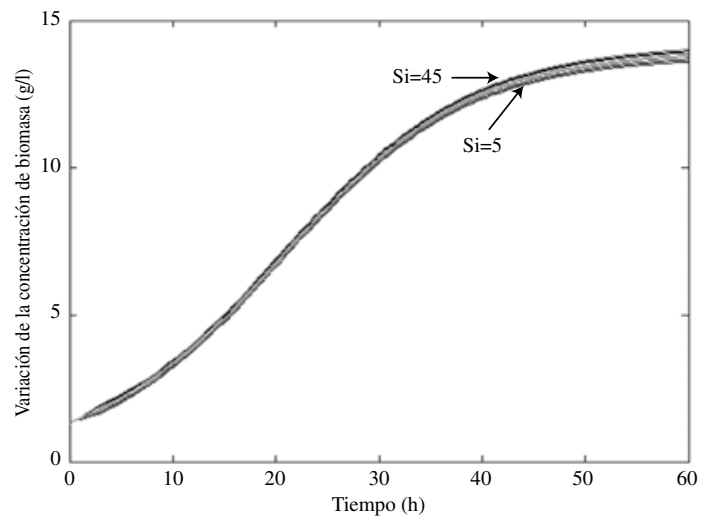

Figura 7. Variación de la concentración de la biomasa ante cambios en la entrada de sustrato.



Figura 8. Variación de la tasa crecimiento específica ante cambios en la entrada de sustrato.

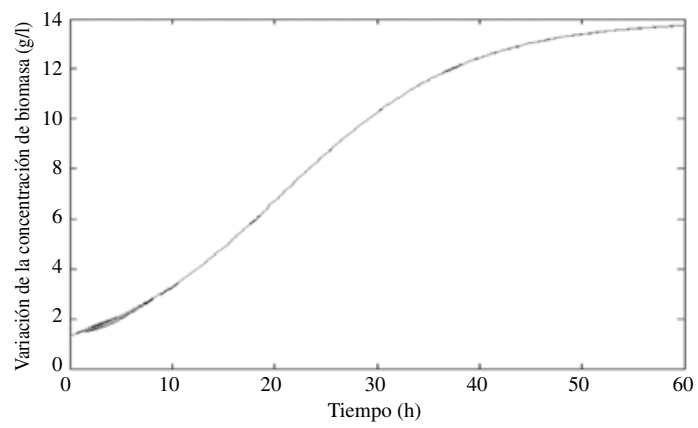

Figura 9. Variación de la concentración de la biomasa ante cambios en la entrada $\lambda$. 


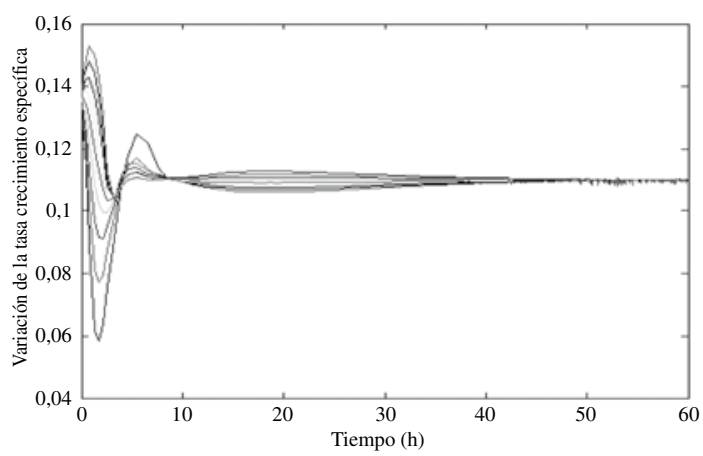

Figura 10. Variación de la tasa crecimiento específica ante cambios en la entrada $\lambda$.

\section{Resultados y análisis del control estabilizante con PI difuso}

Dado que la característica de la concentración de la biomasa ante cambios del sustrato de entrada como de la velocidad de crecimiento varían más para el caso de cambios en lambda $(\lambda)$, se considera cambiar al controlador del lazo del sustrato por un controlador no lineal, como el controlador PI difuso.

Sistema híbrido: PID tradicional en el primer lazo de control con los parámetros proporcional integral presentados en la Tabla 2, y un PI difuso para el lazo de control del sustrato de entrada.

El controlador difuso es del tipo Mamdani, cuyas entradas son: el error de regulación y su primera diferencia, ponderadas por las ganancias $G e$ y $G d e$, respectivamente; y la salida es el incremento del control $u(t)$ normalizado, ponderada por la ganancia $G u$, dando como resultado la variable de control en el lazo de control del sustrato. Se definieron funciones de pertenencia triangulares para las entradas y la salida distribuidas uniformemente en el intervalo [-1,1]. Los parámetros seleccionados para el regulador son: $G e=2 G d e=17$ y $G u=100$. El método de defusificación, utilizado para el controlador difuso normalizado, es la Bisectriz, que ofrece un comportamiento suave y preciso.

Para determinar la efectividad del control se realiza una prueba de robustez de las salidas controladas al variar las entradas del sistema: (i) se hace variar la entrada de sustrato y se mantiene constante $\lambda$ en su valor nominal, de la Figura 11, se aprecia que la concentración de la biomasa tiene un comportamiento de acuerdo a lo deseado por el sistema de referencia, pero con pequeñas variaciones que afectarían al producto final, y, en la Figura 12, la velocidad de crecimiento tiene una desviación mayor al comienzo de la reacción, pero no afecta en la dinámica del producto final; y (ii) las Figuras 13 y 14 muestran las características de la concentración de la biomasa ante un cambio en $\lambda$, considerando constante el sustrato, donde la Figura 13 muestra una pequeña variación solo al comienzo como la observada en la Figura 9.

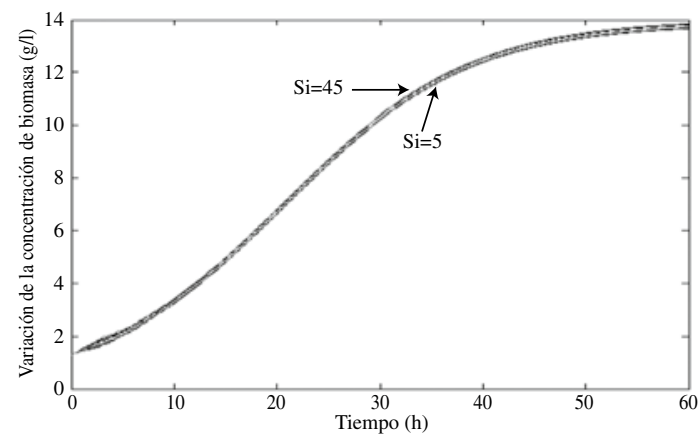

Figura 11. Variación de la concentración de la biomasa ante cambios en la entrada de sustrato.

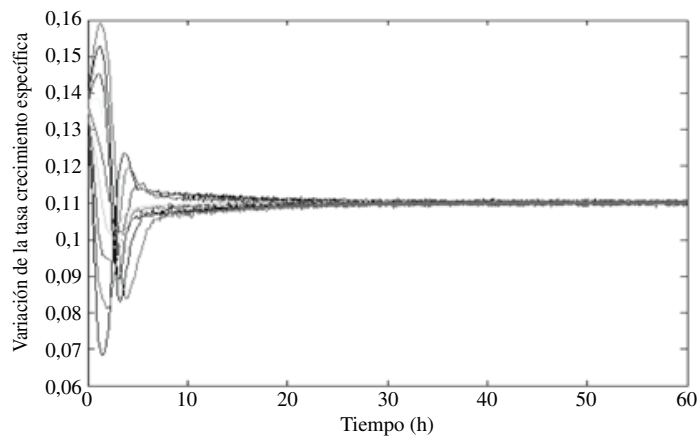

Figura 12. Variación de la tasa crecimiento específica cambios en la entrada de sustrato, $5 \leq s_{i} \leq 45$.

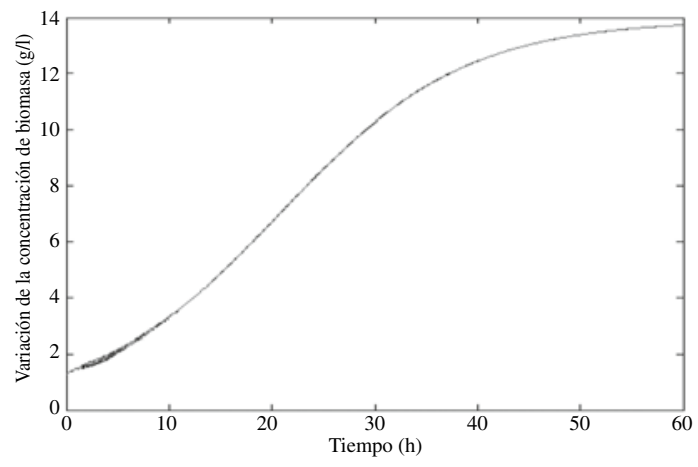

Figura 13. Variación de la concentración de la biomasa ante cambios en la entrada $\lambda$. 




Figura 14. Variación de la tasa crecimiento específica ante cambios en la entrada $0,002 \leq \lambda \geq 0,0016$.

\section{Comparación desempeño controlador difuso v/s NCD-PID}

De acuerdo a las gráficas es muy difícil determinar la efectividad de los controladores experimentados; se sugiere medir desempeño, con respecto a sus valores nominales, según el error cuadrático (RMSE) entre las salidas del sistema: a valores nominales $\mathrm{v} / \mathrm{s}$ variaciones del sustrato y $\lambda$, respectivamente.

La Figura 15 muestra el RMSE de salida de biomasa ante cambios en la entrada de sustrato; el error aumenta a medida que el sustrato de entrada se aleja del valor nominal, además se muestra cómo el control difuso es más robusto ante cambios en la entrada de sustrato. Solo para las entradas $S=20$ y $S=25$ presentan un desempeño aproximadamente igual.

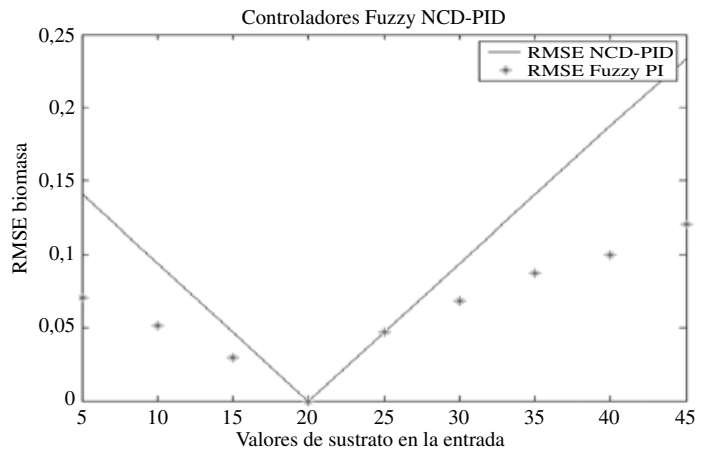

Figura 15. Difuso v/s NCD-PID, valor RMSE de salida biomasa ante cambios en la entrada de sustrato, $S_{i}[\mathrm{~g} / \mathrm{l}]$.
La Figura 16 muestra la medida RMSE de la salida de biomasa ante cambios en la entrada $\lambda$, en esta gráfica se observa que el controlador NCD-PID tiene mejor desempeño que el controlador difuso.

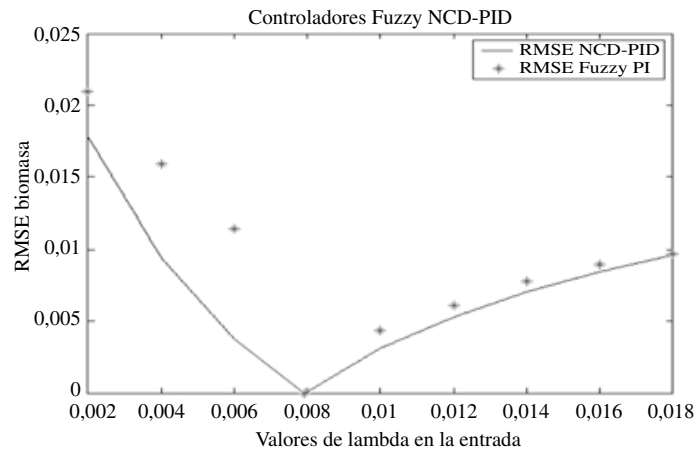

Figura 16. Difuso v/s NCD-PID, valor RMSE de salida biomasa ante cambios en la entrada $\lambda$.

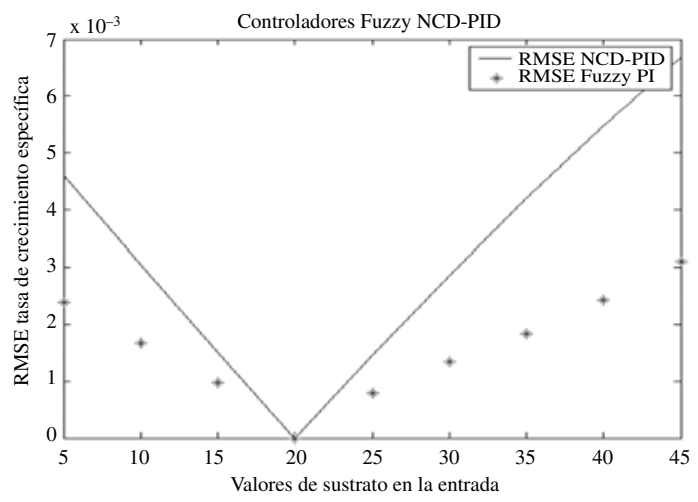

Figura 17. Difuso v/s NCD-PID, valor RMSE de la tasa crecimiento específica ante cambios en la entrada de sustrato.

En la Figura 17 se representa la medida RMSE de la salida para la velocidad de crecimiento específica ante cambios de sustrato de entrada; ella muestra cómo el error aumenta a medida que se aleja del valor nominal, también se aprecia cómo el control difuso es más robusto para todos los valores.

La Figura 18 entrega la medida RMSE de salida de la velocidad de crecimiento específica ante cambios en la entrada $\lambda$, mostrando cómo el controlador difuso se comporta con mayor grado de robustez en un rango más amplio de valores de $\lambda$; sin embargo, el controlador NCD-PID tiene mejor desempeño que el controlador difuso para los valores de $\lambda=0,006$, $\lambda=0,01$ y dentro de estos niveles de $\lambda$. 


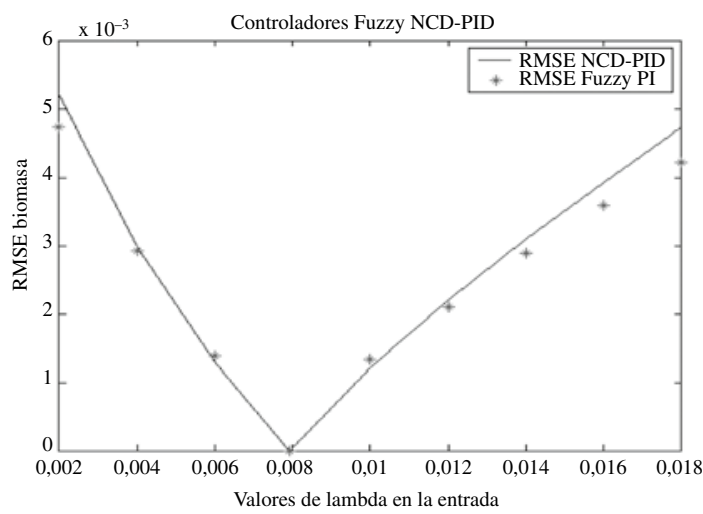

Figura 18. Difuso v/s NCD-PID, valor RMSE de la tasa crecimiento específica ante cambios en la entrada $\lambda$.

Finalmente, la implementación híbrida (Control PI difuso en un lazo de control y PI tradicional en el otro lazo) del PI difuso en el control estabilizante tuvo mejores resultados que el control estabilizante que usa dos PID's (PI tradicional y NCD - PID). Bajo las mismas variaciones de las entrada, se obtuvieron rangos de variaciones de las salidas menores, solo en la Figura 16 se aprecia que el controlador con los PID's tuvo mejor desempeño. En general, el controlador PI difuso resultó ser un gran aporte a la robustez del controlador estabilizante.

\section{CONCLUSIONES}

Se logró encontrar modelos estándares de biorreactores, que involucran un bajo número de ecuaciones y de variables de estado.

El diseño de la ley de control invariante, vía simulación, se aprecia que cumple con mantener la tasa de crecimiento específica constante, con lo cual se obtiene entonces una curva de crecimiento de biomasa aproximadamente exponencial (para una fermentación en modo fed-batch). Consecuentemente se logra fijar la concentración de sustrato en el reactor y se cumple que el volumen crezca ilimitadamente. También se cumple el objetivo de lograr un control con un mínimo de variables medidas (biomasa y volumen solamente). Como el control invariante no logra que las salidas se estabilicen en una referencia determinada (ante variaciones en las entradas), se utilizaron dos métodos de control para el lazo de control de biomasa: el primero fue el controlador NCD-PID llamado así porque se utilizó el Toolbox
NCD para control no lineal, el segundo fue el controlador PI difuso y para el lazo de control de volumen se mantuvieron los parámetros que están en la Tabla 2.

\section{AGRADECIMIENTOS}

A la Dirección de Investigación de la "Universidad de La Frontera" por el financiamiento del Proyecto DIUFRO DI07-0102.

\section{REFERENCIAS}

[1] E. Picó-Marco. "Nonlinear robust control of biotechnological processes. Application to fed-batch bioreactors". Tesis para optar al grado de doctor. Departament d'Enginyeria de Sistemes i Automàtica. Universitat Politècnica de València. España. December 30, 2003.

[2] F.E. Ochoa y V.E. Santos. "Revisión Cinética de transformaciones usando microorganismos I: Modelos cinéticos no-estructurados". Anales de Química. 1994.

[3] G. Bastin and D. Dochain. "On-line estimation and adaptive control of bioreactors". Elsevier. 1990.

[4] I.J. Dunn, E. Heinzle, J. Ingham, J.E. Prenosil. "Biological reaction engineering. Dynamic modelling fundamentals with simulation examples". Second Edition. Wiley-VCH Verlag. 2003.

[5] J.M. Modak, H.C. Lim and Y.J. Tayeb. "General characteristics of optimal feed rate profiles for various fed-batch fermentation processes". Biotechnology and bioengineering. Vol. 28, Issue 9, pp. 1396-1407. 1986.

[6] J. Staniskis and D. Levisauakas. "An adaptive control algorithm for fed-batch culture". Biotechnology and bioengineering. Vol. 26, Issue 5, pp. 419-425. 1984.

[7] K. Schugerl, K.H. Bellgardt. "Bioreaction engineering. Modeling and control". First Edition. Springer-Verlag. 2000.

[8] A.L. Fradkov, I.V. Miroshnik and V.O. Nikiforov. "Nonlinear and adaptive control of complex systems". Kluwer. 1999.

[9] B. Sonnleitner. "Instrumentation of biotechnological processes". Advances in biochemical, Engineering Biotechnology. Vol. 70. 2000. 